\title{
On the place of Parachi and Ormuri among the Iranian languages according to the data of annotated Swadesh lists ${ }^{1}$
}

\begin{abstract}
In this paper, I discuss the open issue of the phylogenetic position of Parachi and Ormuri among other Iranian languages. Based on data from freshly constructed annotated 110-item Swadesh lists of Parachi and Ormuri compared with the same list as tentatively reconstructed for the Proto-Iranian stage (including additional synonyms with Western or Eastern areal distribution), I conclude that Parachi and Ormuri more probably align with the Eastern Iranian branch of languages. This is primarily concluded from the fact that Parachi and Ormuri show correlations with secondary synonyms of Eastern origin. It is important to note that phonetic isoglosses are not nearly as indicative, since Parachi and Ormuri show both Western and Eastern peculiarities; however, the prosodic systems of these languages are strongly reminiscent of the one attested in Pashto and preserving the Proto-(Indo)-Iranian system better than any other Iranian language. These conclusions support Morgenstierne's original opinion on the Eastern character of Parachi and Ormuri; on the other hand, Morgenstierne's assumption of the existence of a separate Southeastern Iranian group requires additional confirmation.
\end{abstract}

Keywords: Parachi language, Ormuri language, 110-item Swadesh list, Proto-Iranian, Eastern Iranian languages, Western Iranian languages, phonetic isoglosses, prosodic systems, Pashto language.

Parachi and Ormuri are two minor Iranian languages which have always presented a significant problem for the internal phylogenetic classification of the Iranian group, with different scholars expressing different opinions regarding their position among other Iranian languages. Thus, G. Morgenstierne thought that Parachi and Ormuri belonged to the Southeastern Iranian group (Morgenstierne 1926: 27-36). On the one hand, he did concede that there were some sound changes common for Parachi, Ormuri and Western Iranian languages, emphasizing that "Parachi and W. Ir. have initial voiced stops $(b, d, g)$ and the palatal affricate $\check{j}_{\text {, }}$ unlike the eastern dialects which have fricatives $(\beta(v), \delta, \gamma, \check{z}$ )" (Morgenstierne 1926: 28; Morgenstierne 1929: 8). On the other hand, he listed numerous examples of Parachi and Ormuri words having specific Eastern Iranian correspondences (Morgenstierne 1926: 31-33; Morgenstierne 1929: 8-12; 317-318).

The opposite opinion was first expressed by G. A. Grierson who wrote that Ormuri "agrees generally, and closely, with the Western Iranian dialects, including Kurdish" (Grierson 1918: 49-52). Similar views were presented in Tedesco 1921 and Oranskij 1979: 81-128. The most extensive argumentation is presented in the monograph Efimov 1986, recently translated into English (Efimov 2011) ${ }^{2}$. According to V. A. Efimov (Efimov 1986: 9; Efimov 2011: 5-6), the following reasons should lead us to consider that Parachi and Ormuri are Western, rather than Eastern, Iranian languages:

1 I would like to thank Dr. Ilya Yakubovich for many valuable helpful comments and remarks. All remaining errors are my own.

${ }^{2}$ For the sake of convenience I cite the English translation throughout this article. 
1) Ir. ${ }^{*} b->$ Orm. $b$-;

2) Ir. ${ }^{*} d->$ Orm. $d-$;

3) Ir. ${ }^{*} g->$ Orm. $g-$;

4) Ir. ${ }^{*}-\check{c}>$ Orm. $\check{z} / z(\log . \check{z}$, Kan. $z)$.

Furthermore, Efimov adduces certain reflexes that fit with the positioning of Ormuri in the Northwestern subgroup of Western Iranian: "the reflexes of Old Iranian ${ }^{*} z{ }^{*} d,{ }^{*} s /{ }^{*} \vartheta$ and ${ }^{*} s p$ corresponding with Ormuri $z, s, s p$, respectively" and "the reflex of Ir. ${ }^{*} \vartheta r /{ }^{*} \vartheta r$ as Log. $\check{s}$, Kan. $\check{r}$ " (Efimov 1986: 9-10; Efimov 2011: 6-7).

The view of Morgenstierne is accepted, for example, in Kieffer 1989 and Kieffer 2009. However, it is important to note that Ch. M. Kieffer is not that optimistic about Morgenstierne's hypothesis in the first work: he mentions both Morgenstierne's and Efimov's views noting Efimov's “riche argumentation" (Kieffer 1989: 452). Efimov's view is accepted in EDIL where both Parachi and Ormuri forms are listed among the data of the Northwestern Iranian languages.

It is clear that Efimov takes into consideration exclusively those isoglosses that concern segmental phonetics. However, there is an additional important trait that is common for Parachi, Ormuri and Eastern Iranian, namely, traces of the Proto-Iranian accentuation, preserved in Pashto and some other Eastern Iranian languages (for example, in Wakhi and Yidgha-Munji languages; see Mayrhofer 1989: 13).

Parachi and Ormuri have free dynamic stress, mobile in the former language and nonmobile in the latter. Efimov characterizes the stress of pre-Ormuri in the following way: "However, as with the Old Iranian dialect to which Ormuri can be traced back, its position is not determined by counting back the long and short syllables from the end of the original word form, that is to say, it does not depend on quantitative factors. From analysis of the material available it can be postulated with sufficient confidence for the original, Old-Iranian dialect that there was a Vedic type of free stress, the reflexes of which may be observed in both the verb and noun systems in varying degrees, although in a more limited and sometimes altered form" (Efimov 2011: 90). This characteristic immediately reminds of Pashto stress, researched in detail by V. A. Dybo in two articles (Dybo 1974; Dybo 1989). Dybo has demonstrated that Pashto inherited free Indo-Iranian stress and preserved some traits of it even better than Vedic. Therefore, it would be very important to investigate the Parachi and Ormuri systems of accentuation and their connection to systems of Pashto and other Eastern Iranian languages that preserve to some extent traces of free stress. It seems that at least Ormuri barytone nouns generally correspond to barytone nouns in Pashto and the same rule is applicable to oxytone nouns, as suggested by the following matches:

1) barytona:

Pashto áspa f. 'horse' Ormuri Kan. yāsp Log. yåsp, f. yấspa 'horse' Vedic áśvā- 'horse' (Dybo 1974: 72; Efimov 2011: 90);

Pashto wấwra 'snow' Ormuri Kan. yoř Log. yoš 'snow' Vedic vápra 'mound; earthwork, rampart, mud wall' (Dybo 1974: 74; Efimov 2011: 90);

Pashto wáč f. wáča 'dry' Ormuri Kan. wyok Log. wuk 'dry' Vedic śúșka- 'dry’ (Dybo 1974: 74; Efimov 2011: 90);

2) oxytona:

Pashto muryá, margá 'bird (of prey)' Ormuri Kan. mirgá Log. morgá 'bird / little bird / sparrow' Vedic mrgá- 'a forest animal or wild beast, game of any kind, (esp.) a deer, fawn, gazelle, antelope, stag, musk-deer' (Dybo 1974: 82; Efimov 2011: 90);

Pashto plấr 'father' Ormuri Kan. pye piyé Log. pe 'father' Vedic pitár- 'father' (Dybo 1974: 74; Efimov 2011: 90); 
Pashto nwí, niwí, nowí ‘90' Ormuri Kan. Log. nawí '90’ OInd. navatí (Dybo 1974: 85; Efimov 2011: 292).

Such correspondences are hardly coincidental, which means that Parachi and Ormuri share at least one very important Eastern Iranian phonetic feature. In order to ascertain its importance for Iranian phylogeny, and also in light of the conflicting scholarly views on the role of phonetic isoglosses in the classification of Parachi and Ormuri on the whole, it seems reasonable to supplement phonetic data with a detailed analysis of the lexical material of the languages under investigation.

To do this, we will recur to lexicostatistical methodology and test the two conflicting hypotheses with the aid of 110-item Swadesh lists for Parachi and Ormuri, contrasting them with the same list as tentatively reconstructed for the Proto-Iranian stage. The methodology of the proto-list reconstruction is described in (Starostin 2016).

For the sake of additional accuracy, the Proto-Iranian list as used in this study includes not only the main ("optimal”) entry, but also other (usually less likely) candidates with indications of their areal distribution. After that, the data of Parachi and Ormuri (which, since the classification of these languages is a priori uncertain, was not used itself for the reconstruction of the Proto-Iranian wordlist in order to avoid circularity) is compared with the reconstructed Proto-Iranian list in order to discover whether these two languages have more specific lexical correlations with Western or Eastern Iranian.

I prefer such a methodology over the more standard lexicostatistical method (comparing data from modern and historically attested languages) for several reasons. First, at this moment we do not have a sufficient number of high-quality annotated Swadesh lists, agreeing with the specifications of the Global Lexicostatistical Database, for Iranian languages (see existing lists at http://starling.rinet.ru/cgi-bin/response.cgi?root=new100\&morpho=0\& basename=new100 \ier $\backslash$ irn\&limit=-1). Second, it is per se interesting to establish the number of specific Western and Eastern isoglosses contained in the list of Proto-Iranian and whether it is possible to make conclusions about the affiliation of Parachi and Ormuri using these data.

The complete data are presented below. The main sources for the Proto-Iranian reconstruction and the list of proto-forms are EDIL and Sergei Nikolaev's Indo-European etymological database included into The Tower of Babel project (http://starling.rinet.ru). The main sources for the Parachi list are G. Morgenstierne's texts and vocabulary (Morgenstierne 1929) and V. A. Efimov's grammar with texts and vocabulary (Efimov 2009). The main sources for the Ormuri list are G. Morgenstierne's Logar texts and vocabulary, including words from both the Logar and Kaniguram dialects (Morgenstierne 1929), V. A. Efimov's Kaniguram and Logar texts and grammar with etymological vocabulary (Efimov 2011) and D. G. Hallberg's 210-item wordlist for Kaniguram partly coinciding with Swadesh lists (Hallberg 2004). Based on these data, it is possible to make some observations on the Parachi and Ormuri lists and count areal isoglosses.

First, it is interesting that Kaniguram and Logar dialects show numerous differences in both inherited and borrowed lexicon. The following cases are attested:

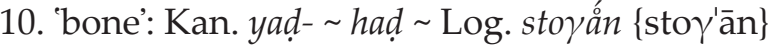

30. 'fly (v.)': Kan. bur- / bur-yék Log. par- / par-ók

44. 'knee': Kan. zan-řrak / zān-řrak Log. zånú $\{z a \bar{n} n \bar{u}\}$

46. 'leaf': Kan. puxay Log. barg

54. 'moon': Kan. maryok / Kan. spožmay Log. måtáw

72. 'see': Kan. dzun- Log. ǰŭ-

75. 'skin': Kan. tsarmun / čāmra Log. pōst 
78. 'smoke': Kan. lugay Log. dud $\{$ dūd $\}$

79. 'stand': Kan. ašt- / ašt-yék / Kan. t-yek Log. dar / dar-ók

90. 'tree': Kan. wúna Log. draxt

101. 'far': Kan. pets Log. dur

102. 'heavy': Kan. grān Log. wazmînd

106. 'snake': Kan. māngor Log. mår.

It must, however, be noted that some of these discrepancies may be fictitious due to lack of sufficient information in our sources - it is possible that some of the loanwords are actually used in both dialects, or that one dialect uses not one but many borrowings as synonyms.

Second, there are 3 exclusive Parachi-Ormuri isoglosses in the 110-item wordlist:

1. 'all': Parachi hu / hos Ormuri (Kan., Log.) ayéra < PIr. *harwa (EDIL 3: 372-373).

19. 'drink': Parachi t'er- / t'ar- / tōor- Ormuri (Kan., Log.) tr- / ta-tak / tō-tk< PIr. ${ }^{*}$ tr-ya- / *tršta(ka)- (Morenstierne 1929: 10, 295).

28. 'fire’: Parachi r'ínē Ormuri (Kan.) ráwan, (Log.) rówon < PIr. *rauxšna- (Morgenstierne 1929: 284; Efimov 2011: 296).

Although this number seems to be rather small, it is important to remember that both languages have a significant number of borrowings and lexicographic lacunae; moreover, sometimes it is possible to find such exclusive non-lexicostatistical pairs with divergent meanings as Ormuri gap 'stone' Parachi gáp-âr 'fireplace' (Morgenstierne 1929: 10). The natural conclusion is that these two languages really form one subgroup, going back to an intermediate ancestor.

Finally, the number of areal isoglosses in the Proto-Iranian wordlist is significant. The following 15 isoglosses define the Western group:
4. 'belly’: SW *škamb-, NW*lab-.
7. 'bite': SW, NW ${ }^{*}$ gaz-.
11. 'breast': SW, $\mathrm{NW}^{*}$ cai-na-.
26. 'fat': SW, NW *čarp-īh-.
36. 'hair': SW, NW *maud-a-.
43. 'kill': SW, NW ${ }^{*} k a u s ̌-$.
55. 'mountain': SW, NW *kaufa-.
56. 'mouth': SW, NW *3af-an-.
61. 'nose': SW, NW *wain-a- *wain-i-.
70. 'sand': SW, NW *raika-.
71. 'say': SW, NW *gaub-.
104. 'salt': NW *hwād-.
106. 'snake': SW, NW *mār-a-.
107. 'thin': SW, NW *nāz-u-ka-.

Two of these isoglosses are specifically Northwestern, one specifically Southwestern; all the others are common to both branches.

There are 10 Eastern isoglosses in the Proto-Iranian list:

5. 'big': *stūra-

12. 'burn': *Oaw-

15. 'cold': *stāba-

18. 'dog': *kuta- / fem. *kuti( $(\bar{\imath})-$

26. 'fat': *wāzdā-

29. 'fish': *kapa- 
35. 'green *ax̌̌aina-

36. 'hair': *gaun-a-

93. 'warm': *tap-

106. 'snake': *Savio $\gamma c$ - (the Proto-Shughni form; Morgenstierne 1974: 30).

It seems clear that Parachi and Ormuri do not generally share Western isoglosses. There is only one clear case: Parachi gas- / gast- / gōst- 'to bite / to sting' is derived from *gaz- 'to bite / to sting' (EDIL 3: 263-265; a related word in Pashto is ayzáy 'thorn', see Cheung 2007: 118, but the verb itself is not attested in Eastern Iranian).

In contrast, Parachi and especially Ormuri do share several Eastern innovations (and this result fully agrees with Morgenstierne's lexical observations).

Parachi shows 3 matches with Eastern Iranian:

12. 'burn': Parachi tēê- / thēyí EIr. * $\theta a w-;$

26. 'fat': Parachi $\gamma a z d \sim$ EIr. ${ }^{*} w \bar{a} z d \bar{a}-;$

36. 'hair’: Parachi ginố $\sim$ EIr. * gaun-a.

Also worth mentioning is Parachi ta'pö, which at face value is recognizable as a Pashai loanword, but it is also possible that earlier *tapaka- was deformed under Pashai influence; compare Ormuri (Kan., Log.) tok 'warm / hot'.

Ormuri has 4 matches with Eastern Iranian:

5. 'big': Ormuri (Kan.) stur, (Log.) stor EIr. *stūra-;

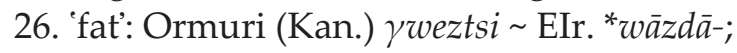

35. 'green': Ormuri (Kan.) šin, (Log.) x̌in EIr. *axšaina-;

93. 'warm': Ormuri (Kan., Log.) tok EIr. *tap-.

My conclusions are based on the theoretical assumption that the Proto-Iranian 110-item Swadesh list is sufficient to demonstrate the affinity of two languages with a concrete subgroup of related idioms, while theories of classification that are based on phonetic isoglosses alone are generally weak and demand lexical support (cf. Dybo, Starostin 2008). If so, then evidence provided by the basic lexicon leads to the natural conclusion that Parachi and Ormuri are not Western (Northwestern) Iranian languages, and that it makes more sense to classify them as Eastern Iranian languages, following Morgenstierne (however, the notion of Southeastern Iranian as a genetic subgroup is controversial and requires independent confirmation).

That said, this result should not be considered as final, and further investigation is necessary to demonstrate the exact degree of relationship between Parachi-Ormuri and other Eastern Iranian languages. I think that there are three main directions in which this investigation should be taken. First of all, it is necessary to increase the number of high-quality annotated Swadesh lists for Iranian languages in order to obtain more precise lexicostatistical matrices and arrive at more refined phylogenetic conclusions. The second task is to revise the lexical isoglosses assembled by Morgenstierne and add new data gathered from text corpora and etymological dictionaries. It would also be useful to conduct a thorough analysis of the relatively scarce specific correspondences between Parachi-Ormuri and Western Iranian subgroups and see whether they can be explained as cases of accidental homoplasy or results of contact-induced change.

Finally, a third direction of further studies - arguably the most intriguing one for specialists not only in Iranian, but also in general Indo-European studies - should consist of research on the prosodic systems in Parachi and Ormuri and their relation to the corresponding systems in Pashto and other Eastern Iranian languages. 


\section{Appendix 1. 110-item Swadesh lists for Proto-Iranian, Parachi and Ormuri.}

Preliminary note: I mostly use Efimov's transcription for both Parachi and Ormuri. Present and past Parachi verbal stems are cited according to Efimov 2009 (see especially Efimov 2009: 65-71). Lexical synonyms are only given in those cases where available data does not allow to choose the most accurate semantic equivalent for the required Swadesh meaning. Borrowings are marked with negative indexes $(-1)$, with the name of the donor language or language group indicated after the index.

All Ormuri forms are marked as Kan. (Kaniguram) and Log. (Logar), since there are significant differences between two dialects. Three verbal stems are cited consistently: the present stem, masc. sg. past participle, sg. fem. past participle ${ }^{3}$.

An extended annotated version of the Parachi list can be found at the Global Lexicostatistical Database (http://starling.rinet.ru/new100).

\begin{tabular}{|c|c|c|c|}
\hline Word & Proto-Iranian & Parachi & Ormuri \\
\hline 1. all & ${ }^{*} w i c-w-a-$ & $h u \sim h o s$ & Kan. Log. ayéra \\
\hline 2. ashes & *ātr-ya- & jond -1 Ind. & Kan. yānak yā̄ $\eta k$ \\
\hline 2. ashes $_{2}$ & & b’aㄱ -1 Pers. & \\
\hline 3. bark & *pawasta- & pust -1 Pers. & Log. pāṇ̄-1 Psht. ? \\
\hline 4. belly & *udar-a- & aštáw & Kan. dim -1 Dard. \\
\hline 4. belly 2 & *škamb-SW & & Kan. Log. nas -1 Psht. \\
\hline 4. belly 3 & $* l a b-\mathrm{NW}$ & & \\
\hline 5. big & ${ }^{*} m a 3-\sim{ }^{*} m a 3-a n t-\sim{ }^{*} m a 3-a-n a-$ & giand -1 Ind. / Dard. & Kan. stur Log. stor \\
\hline 5. big $_{2}$ & ${ }^{*}$ stūra- EIr & & \\
\hline 6. bird & $w i-$ & not attested & Kan. mirgá Log. morgá \\
\hline 7. bite & *danc- & gas- / gast- / gōst- & Kan. Log. gran- \\
\hline 7. bite $_{2}$ & *gaz-SW NW & & $\begin{array}{c}\text { Kan. } x r-/ x^{w} a ́-l a k / x^{w} \bar{a}-l k \\
\text { Log. } x r-/ x o ́-l o k\end{array}$ \\
\hline 8. black & ${ }^{*} c y \bar{a} w-a-$ & pádō-1?? & Kan. Log. $\gamma r a ̄ s$ \\
\hline 9. blood & ${ }^{*} w a h-u n-i-\sim{ }^{*} w a h-u n-a-$ & hin & Kan. sun Log. šun \\
\hline 9. blood $_{2}$ & & & Log. in -1 Psht. ? \\
\hline 10. bone & ${ }^{*}$ ast $-\sim{ }^{*}$ ast $-i-$ & had -1 Ind. / Dard. & $\begin{array}{c}\text { Kan. yad- had -1 Psht. < } \\
\text { Ind. / Dard. }\end{array}$ \\
\hline 10. bone $_{2}$ & & & 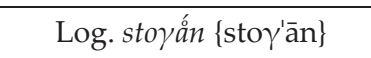 \\
\hline 11. breast & ${ }^{*} w a r-a h-$ & siz & Log. sónok sínak \\
\hline 11. breast $_{2}$ & ${ }^{*}$ cai-na-SW NW & bar -1 Pers. & \\
\hline 12. burn & ${ }^{*}$ dag- & têew- / thēyí & $\begin{array}{c}\text { Kan. braz- / braš-tak / bruš-k } \\
\text { Log. bróx́-tok }\end{array}$ \\
\hline 12. burn $_{2}$ & ${ }^{*} \theta a w-$ EIr (Pamir) & & \\
\hline $\begin{array}{l}\text { 13. claw } \\
\text { (nail) }\end{array}$ & *nāx-u-na- / *nāx-a-na- & nōrk & $\begin{array}{c}\text { Kan. naxk } \\
\text { Log. néxči (sg. and pl.) }\end{array}$ \\
\hline 14. cloud & ${ }^{*} a b r-a-$ & ayír & Kan. abnr Log. yēwar \\
\hline 14. cloud $_{2}$ & ${ }^{*}$ maig-a & & \\
\hline 15. cold (adj.) & ${ }^{*} \mathrm{car}-\mathrm{ta-}$ & ešțátw & Kan. Log. tsak \\
\hline
\end{tabular}

\footnotetext{
${ }^{3}$ The third form is frequently not attested.
} 


\begin{tabular}{|c|c|c|c|}
\hline Word & Proto-Iranian & Parachi & Ormuri \\
\hline 15. cold $_{2}$ (adj.) & ${ }^{*}$ stāba- & & \\
\hline 16. come & ${ }^{*}$ gam- & $\check{z} i-$ & $\begin{array}{c}\text { Kan. } z \text { - z-ay- / z-ok / z-āk } \\
\text { Log. z-ey- / z-ok / z-åk }\end{array}$ \\
\hline 16. come $_{2}$ & & $\stackrel{\circ}{a} \gamma-$ & \\
\hline 17. die & ${ }^{*}$ mar- & mer- / mor- & $\begin{array}{c}\text { Kan. } m r-\text { / mú-lak / ma-lk } \\
\text { Log. mr- / mó-lok }\end{array}$ \\
\hline 18. dog & ${ }^{*}$ cwan- & espó & Kan. spak Log. spok \\
\hline 18. $\operatorname{dog}_{2}$ & ${ }^{*} k u t a-$ / fem. ${ }^{*} k u t i(\bar{\imath})$ - & & \\
\hline 18. $\operatorname{dog}_{3}$ & ${ }^{*} g a d-w-a-$ & & \\
\hline 19. drink & *hwar- & t'er- / t'ar- / t'ōr- & Kan. Log. $t r-$ / ta-tak / tō-tk \\
\hline 19. drink $_{2}$ & ${ }^{*} p \bar{\imath}-/{ }^{*} p \bar{a}-$ & & \\
\hline 20. dry & *huš-ka- & hóškō & Kan. wyok Log. wuk \\
\hline 21. ear & ${ }^{*}$ gauš-a- & guš & Kan. Log. goy \\
\hline 22. earth & ${ }^{*} 3 a m-$ & d'arám -1 Ind. / Dard. & $\begin{array}{l}\text { Log. búma bóma } \\
\text { Kan. búma bumba }\end{array}$ \\
\hline 22. earth 2 & & & Log. ǰer \\
\hline 23. eat & *hwar- & xar- / xur- & $\begin{array}{c}\text { Kan. } x r-/ x^{w} a ́-l a k / x^{w} \bar{a}-l k \\
\text { Log. } x r-/ x o ́-l o k\end{array}$ \\
\hline 24. egg & ${ }^{*} \bar{a} i-a-\sim * \bar{a} i-a-k a-$ & $\bar{e} x$ & $\begin{array}{c}\text { Kan. älk hãwalk wonk } \\
\text { Log. wolk }\end{array}$ \\
\hline 25. eye & *čaš-man- & teč & $\begin{array}{c}\text { Kan. tsom } \\
\text { Log. tsími (sg. and pl.) }\end{array}$ \\
\hline 26. fat & ${ }^{*} p \bar{\imath}-w a h-$ & čarbí -1 Pers. & Kan. $\gamma w e z t s i$ \\
\hline 26. fat $_{2}$ & *čarp-īh-SW NW & yazd & \\
\hline 26. fat $_{3}$ & ${ }^{*} w \bar{a} z d \bar{a}-\mathrm{EIr}$ & & \\
\hline 27. feather & *par-na- & pōn & Kan. púxay (pl.) \\
\hline 27. feather 2 & & piar-1 Pers. & \\
\hline 28. fire & ${ }^{*} \bar{a}$-tar- & $a ̊$ & Kan. ráwan Log. rówon \\
\hline 28. fire $_{2}$ & & rinne & \\
\hline 29. fish & ${ }^{*}$ macy-a- $\left(<{ }^{*}\right.$ matsy-a- $)$ & miásō & Kan. Log. māyí -1 Pers. \\
\hline 29. fish $_{2}$ & *kapa- & & \\
\hline 30. fly v. & *pat- & råz- & Kan. bur- / bur-yék \\
\hline 30. fly v. & & p'arak- -1 Pers. ? & Log. par- / par-ók -1 Pers.? \\
\hline 31. foot & *pad- & $p a ̊$ & Kan. pá́-ri Log. påy \\
\hline 32. full & *pr-na- & tiad & Kan. dāk Log. dåk -1 Psht. \\
\hline 33. give & ${ }^{*} d \bar{a}-$ & dah- / då- & $\begin{array}{c}\text { Kan. řáw-/ řyuk / řuk } \\
\text { Log. šáw- / šuk }\end{array}$ \\
\hline 34. good & *wah-u- & bakár -1 Pers. & Kan. sir $\sim \operatorname{sirr}$ Log. šer \\
\hline 35. green & ${ }^{*} z a r-i-$ & sawz -1 Pers. & Kan. šin Log. x̌in \\
\hline 35. green $_{2}$ & *axšaina- EIr & & \\
\hline 36. hair & ${ }^{*} w a r c-a-$ & ginó & $\begin{array}{l}\text { Kan. dra / pl. dri } \\
\text { Log. dri (sg. \& pl.) }\end{array}$ \\
\hline 36. hair $_{2}$ & ${ }^{*}$ maud-a- & döš & \\
\hline 36. hair $_{3}$ & ${ }^{*}$ gaun- $a-$ & & \\
\hline
\end{tabular}




\begin{tabular}{|c|c|c|c|}
\hline Word & Proto-Iranian & Parachi & Ormuri \\
\hline 37. hand & $*_{3 a s-t a-}$ & dōst & Kan. dist Log. dest \\
\hline 38. head & ${ }^{*}$ car-ah- & sōr $r$ & Kan. Log. sar \\
\hline 39. hear & ${ }^{*}$ craw- & harw- / hat- / hōt- & $\begin{array}{c}\text { Kan. amár- / amar-yék } \\
\text { Log. mar- amar- / marók } \sim \text { amarok }\end{array}$ \\
\hline 40. heart & ${ }^{*} 3 r d-$ & zor & Kan. zli Log. zle $\sim$ zli \\
\hline 41. horn & ${ }^{*} \mathrm{cr}-\bar{u}-$ & šax -1 Pers. & Kan. sukár \\
\hline 41. horn $_{2}$ & & $\check{S}_{\bar{i}}$ & \\
\hline 42. I & *az-am & $\stackrel{\circ}{a n}$ & Kan. Log. $a z$ \\
\hline 42. $\mathrm{I}_{2}$ (obl.) & $m a-$ & mon & Kan. Log. mun \\
\hline 43. kill & *gan- & mêr - / månt- & $\begin{array}{c}\text { Kan. wazn- / wáz-yok } \\
\text { Log. wožn-áw- / wožn-aw-ók }\end{array}$ \\
\hline 43. kill $_{2}$ & *kauš- SW NW & & \\
\hline 44. knee & *3ān-u- & zånú -1 Pers. & 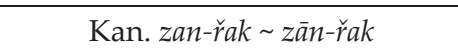 \\
\hline 44. knee $_{2}$ & & & Log. zånú $\{z a ̄ n u ̄ y\}-1$ Pers. \\
\hline 45. know & wid- & xabár bi- -1 Pers. & $\begin{array}{l}\text { Kan. pazán- / pazan-yék } \\
\text { Log. pazán- / pazan-ók }\end{array}$ \\
\hline 45. know $_{2}$ & & & $\begin{array}{c}\text { Kan. poy awas- } \\
\text { Log. poy b- / poy šáw- -1 Psht. }\end{array}$ \\
\hline 46. leaf & *war-ka- & pōn & Kan. puxay \\
\hline 46. leaf & & & Log. barg -1 Pers. \\
\hline 47. lie & ${ }^{*} \mathrm{cai}-$ & riz- & Kan. nw- / nw-ástak \\
\hline 48. liver & *yak-ar / *yak-n- & ǰegár -1 Pers. & Kan. $d z \bar{a} \check{r}$ \\
\hline 49. long & ${ }^{*}$ darg- $a-$ & bišt & Kan. drā Log. drå \\
\hline 50. louse & *cwiš- & espó & Kan. Log. spuy \\
\hline 51. man & *nar- & $m \bar{e} r$ & Kan. Log. saray -1 Psht. \\
\hline 52. many & *par-u- & yolú -1 Pers. < Arab. & Kan. dzut zut Log. zot \\
\hline 53. meat & *gaw- & $\gamma u \check{s}$ & Kan. gấka Log. gåka \\
\hline 54. moon & *māh- & mahốk & Kan. maryok \\
\hline 54. moon $_{2}$ & & & Kan. spožmay -1 Psht. \\
\hline 54. moon $_{3}$ & & & Log. måtáw -1 Pers. ? \\
\hline 55. mountain & ${ }^{*} g a r-i-$ & d'år -1 Dard. / Ind. & Kan. gri Log. gri girí \\
\hline 55. mountain $_{2}$ & *kaufa- & & \\
\hline 56. mouth & ${ }^{*} \bar{a} h-$ & šoṇd -1 Dard. & Kan. pyoz Log. poz \\
\hline 56. mouth $_{2}$ & ${ }^{*} 3 a f-a n-\mathrm{SW}$ NW & & \\
\hline 57. name & *nām-an- & nåm & Kan. nām Log. nåm \\
\hline 58. neck & ${ }^{*}$ grīw-a- & mandá -1 Dard./Ind. & Kan. gardan Log. gardan -1 Pers. \\
\hline 59. new & ${ }^{*} n a v-a-$ & now & Kan. nyow Log. now \\
\hline 60. night & *xšap- & xawán & Kan. šyo Log. $\check{x} o$ \\
\hline 61. nose & ${ }^{*} n a h-/{ }^{*} n \bar{a} h-$ & $n \bar{e} s ̌ t$ & Kan. Log. ninní \\
\hline 61. nose $_{2}$ & ${ }^{*}$ wain- $a-\sim^{*}$ wain- $i-\mathrm{SW}$ NW & & \\
\hline 62. not & ${ }^{*} n a-$ & $n a$ & Kan. Log. nak \\
\hline 63. one & $* a i-w a-$ & $\check{z} u$ & Kan. sa Log. še \\
\hline 64. person & ${ }^{*} m a r-t-y a-/{ }^{*} m a r-t a-$ & månéš -1 Ind. & Kan. Log. saray -1 Psht. \\
\hline
\end{tabular}




\begin{tabular}{|c|c|c|c|}
\hline Word & Proto-Iranian & Parachi & Ormuri \\
\hline 65. rain & *wār $(a)$ & yår & Kan. bārān Log. bårån -1 Pers. \\
\hline 66. red & *raud-i-ta- & sork sórkō & Kan. suř Log. šuš \\
\hline 67. road & 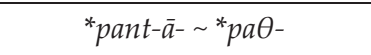 & panán & Kan. rāy, Log. råy \\
\hline 68. root & *waix-a- & $\gamma \bar{i} x$ & Kan. walyi \{walye $\}-1$ Psht. \\
\hline 68. root & ${ }^{*} r a i s ̌-a-k a-$ & & \\
\hline 69. round & ${ }^{*}$ gart-a-na- & not attested & not attested \\
\hline 70. sand & *raika- SW NW & $\begin{array}{c}\text { seyá-1 Ind. / Dard. / Ir. } \\
?\end{array}$ & Kan. siga -1 Psht. \\
\hline 71. say & ${ }^{*}$ mraw- & jar- / jaari:- & Kan. $\gamma^{w} a s-\log . \gamma o s_{-}$ \\
\hline $\begin{array}{l}\text { 71. } \text { say }_{2} \\
\text { (suppl.) }\end{array}$ & *wač- & & \\
\hline 71. say $_{3}$ & ${ }^{*} g a u b-S W N W$ & & \\
\hline 72. see & *wain- & b’oč- -1 Dard. / Ind. & Kan. $d z u n-$ \\
\hline 72. see $_{2}$ & & & Log. $\check{\jmath} u \check{x}-$ \\
\hline $\begin{array}{l}\text { 72. } \text { see }_{3} \\
\text { (suppl.) }\end{array}$ & ${ }^{*}$ darc- & dior- & Kan. dyek Log. dek \\
\hline 73. seed & *taux-man- & poọ -1 Dard. / Ind. & Log. toxam toxom -1 Pers. \\
\hline 74. sit & $* \bar{a} h-$ & n’in- / niašț- / niōšț & $\begin{array}{c}\text { Kan. āy- ey-/ nás-tak } \\
\text { Log. nós-tok }\end{array}$ \\
\hline 75. skin & *pawasta- & pust -1 Pers. & Kan. tsarmun / čāmra -1 Psht. \\
\hline 75. skin $_{2}$ & & & Log. pōst -1 Pers. \\
\hline 76. sleep & *hwap- & xōm kan- & $\begin{array}{l}\text { Kan. } x^{w} \bar{a} w k-/ \text { dok } \\
\text { Log. xaw k- / dåk } \\
\text { Kan. } x^{w} \text { al-gastak }\end{array}$ \\
\hline 77. small & ${ }^{*} k a c-u-$ & činó -1 Dard. & Kan. Log. zarí \\
\hline 77. small $_{2}$ & & ríza-1 Pers. & \\
\hline 78. smoke & ${ }^{*} d \bar{u}-t a-/{ }^{*} d \bar{u}-m a-$ & $d^{\prime} i$ & Kan. lugay -1 Psht. \\
\hline 78. smoke & & & Log. dud $\{\mathrm{d} \overline{\mathrm{u}} \mathrm{d}\}-1$ Pers. \\
\hline 79. stand & *stā- & papá bi- & Kan. ašt- / ašt-yék \\
\hline 79. stand $_{2}$ & & & Kan. t-yek \\
\hline 79. stand ${ }_{3}$ & & & Log. dar / dar-ók \\
\hline 80. star & ${ }^{*}$ stār & estééc & $\begin{array}{l}\text { Kan. stirrak storak } \\
\text { Log. stežáy stéšak }\end{array}$ \\
\hline 81. stone & ${ }^{*} a c-m a n-/{ }^{*} a c-a n-g a-$ & $g \bar{\imath} r$ & Kan. Log. gap \\
\hline 82. sun & *hwar- / "hwan- & ruč & Kan. meř Log. meš \\
\hline 82. $\operatorname{sun}_{2}$ & & & Log. tówa Kan. tōws \\
\hline 83. swim & *fraw- & aubá́zì kan- -1 Pers. & Log. aubāzī k--1 Pers. \\
\hline 84. tail & ${ }^{*} d u m-a-/{ }^{*} d u m-b-a-$ & domb-1 Pers? & Kan. likye -1 Psht. \\
\hline 84. tail & & dayónd & Log. dom \\
\hline 85. that & $\begin{array}{c}\text { *ana- 'that' (medial) / } \\
\text { *haw- (masc., fem.) } \\
\text { *awa- (neut.) 'that' (distal) }\end{array}$ & $\bar{o}$ & Kan. afa $\sim$ afo Log. afo \\
\hline 86. this & $\begin{array}{c}{ }^{*} a i-\text { (masc.) } \sim \\
*_{i-}(\text { fem., neut.) 'this' }\end{array}$ & $\bar{e}$ & Kan. $o$ Log. $a$ \\
\hline 87. thou & ${ }^{*} t \bar{u}-\sim^{*} t u w-a m$ & tō & Kan. Log. $t u$ \\
\hline
\end{tabular}




\begin{tabular}{|c|c|c|c|}
\hline Word & Proto-Iranian & Parachi & Ormuri \\
\hline 88. tongue & ${ }^{*} h i z-\bar{u}-$ & bån & Kan. zbān Log. zobán \{zu'bān\} \\
\hline 89. tooth & ${ }^{*}$ dant- $\sim{ }^{*}$ dant-an- & danắn & Kan. gas Log. gíši (sg. and pl.) \\
\hline 90. tree & *dār-u- & bin & Kan. wúna -1 Psht. \\
\hline 90. tree $_{2}$ & & & Log. draxt -1 Pers. \\
\hline 91. two & $* d w a-$ & $d i \sim d o$ & Kan. dyo Log. do \\
\hline 92. walk (go) & ${ }^{*} a i-$ & par- & $\begin{array}{l}\text { Kan. tsaw- / ts-yek } \\
\text { Log. tsaw- / tsaw-ok }\end{array}$ \\
\hline $\begin{array}{l}\text { 92. walk (go })_{2} \\
\text { (suppl.) }\end{array}$ & & $\check{c} i-$ & \\
\hline 93. warm (hot) & ${ }^{*}$ gar-ma- & ta'pö-1 Dard. & Kan. Log. tok \\
\hline 93. warm (hot) $)_{2}$ & ${ }^{*}$ tap- EIr & & \\
\hline 94. water & ${ }^{*} \bar{a} p-/{ }^{*} a p-$ & $\stackrel{a}{a} w$ & Kan. wak Log. wok \\
\hline 95. we & *wai-am & $m a ̊$ & Kan. māx Log. måx \\
\hline $\begin{array}{l}\text { 95. } \text { we }_{2} \\
\text { (suppl.) }\end{array}$ & *ah-ma- & & \\
\hline 96. what & ${ }^{*} k a-t$ & $\check{c} e$ & Kan. Log. tsa \\
\hline 97. white & ${ }^{*}$ cwait-a- ${ }^{*}$ cwit- $a-$ & čćáčō-1 Dard. / Ind. & Kan. spew spiw Log. spew \\
\hline 98. who & ${ }^{*} k a-$ & $k a$ & Kan. kuk Log. kok \\
\hline 99. woman & 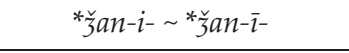 & zaíf-1 Pers. < Arab. & Kan. dzarká Log. zarká \\
\hline 100. yellow & ${ }^{*} 3 a r-i-$ & zítö & Kan. Log. zyer -1 Psht. \\
\hline 101. far & ${ }^{*} d \bar{u} r-a i$ & dorín & Kan. pets \\
\hline 101. far $_{2}$ & & & Log. dur -1 Pers. ? \\
\hline 102. heavy & ${ }^{*} g u r-u-$ & gerån -1 Pers. & Kan. grān \\
\hline 102. heavy 2 & & beån-1 Ind. & Log. wazmínd -1 Pers. \\
\hline 103. near & *nazd- & nazdík -1 Pers. & Kan. Log. boy \\
\hline 103. near $_{2}$ & & & Log. nezdék \\
\hline 104. salt & *namada-ka- *namad-ka- & namá & Kan. mek Log. nemék \\
\hline 104. salt & *hwād- NW & & \\
\hline 105. short & ${ }^{*} m r 3-u-$ & ránōk & Kan. Log. land -1 Psht. \\
\hline 106. snake & $* a \check{5}-i-$ & kerm -1 Pers. & Kan. māngor -1 Psht. \\
\hline 106. snake ${ }_{2}$ & *mār-a-SW NW & & Log. mår -1 Pers. \\
\hline 106. snake $_{3}$ & ${ }^{*} \delta \partial v u ̛ \gamma c-($ Shughni) & & \\
\hline 107. thin & ${ }^{*} \tan -u-k a-$ & mahín-1 Pers. & Kan. narye Log. naráy -1 Psht. \\
\hline 107. thin ${ }_{2}$ & ${ }^{*} n \bar{a} z-u-k a-$ & & \\
\hline 108. wind & $* w \bar{a}-t a-$ & $\gamma \bar{a}\{\gamma \hat{a}\}$ & Kan. $b \bar{a} d-1$ Pers. \\
\hline 108. wind 2 & & šamál -1 Pers. < Arab. & \\
\hline 109. worm & ${ }^{*} k r m-i-$ & kerm -1 Pers. & Log. kerm -1 Pers. \\
\hline 110. year & *yār- & sar & Kan. tsān Log. čån \\
\hline
\end{tabular}

Notes on specific Swadesh items.

1. 'all': Proto-Iranian: * $w i c-w-a$ - is the best candidate: its descendants mean 'all' in Avestan and in some Middle Iranian languages (like Sogdian). In many Iranian languages (both Western and Eastern) *wic-w- $a$ - was superseded by the stem *ham-a- with the original meaning 'the same'; in Parachi and Ormuri it has been replaced by reflexes of *harw- $a$ - 'whole (totus)'. 
3. 'bark': Proto-Iranian: *pawasta- is likely to be analyzed as the past participle ${ }^{*} p a=w a s-t a-$ from the verbal root*was- 'to wear clothes, be dressed' with the prefix *(a)pa-. The first meaning of this form is 'skin'; polysemy 'skin / bark' is found in many Iranian languages and can be reconstructed for the proto-language.

4. 'belly': Proto-Iranian: *udar-a- is retained in Avestan, Sogdian and in many Eastern Ira-

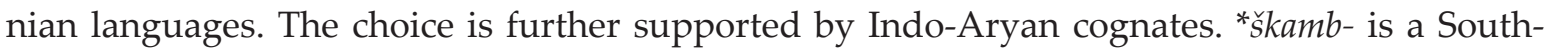
western isogloss; ${ }^{*} l a b$ - is a Northwestern isogloss.

Parachi: Probably of Indo-Iranian origin.

5. 'big': Proto-Iranian: The root ${ }^{*}$ maz- and its derivatives are retained in Avestan, Sogdian, Southwestern, Northwestern (Kurdish mazin, Balochi mazan) and in some Eastern Iranian languages (for example, Manichean Sogdian $m z y \gamma<{ }^{*} m a z-i k-a$, a secondary formation of the comparative grade *maz-iyah). It has a reliable Indo-European etymology and exact matches in Old Indic. ${ }^{*}$ stūra- is attested in this meaning in Eastern Iranian languages.

6. 'bird': Proto-Iranian: The stem *wi- is retained in Avestan and Middle Persian and is supported by Vedic evidence. In many Iranian languages, 'bird' is expressed with reflexes of *mrga-.

Parachi: It is possible to suggest that the main word for 'bird' is morče 'sparrow' (Efimov 2009: 218; Morgenstierne 1929: 273) (like in Ormuri, see below), but this is uncertain.

Ormuri: Though this word is usually translated as 'sparrow / little bird', Hallberg's informants use it to translate phrases "the man killed (shot) the bird" and "the bird flew" (Hallberg 2004: 141). It means that Ormuri shows polysemy 'sparrow / little bird / bird in general'.

7. 'bite': Proto-Iranian: The stem *danc- is retained in Avestan and Khotanese Saka and is supported by Indo-Aryan cognates. ${ }^{*} g a 3^{-}$is attested in the meaning 'to bite / to sting' in Southwestern and Northwestern Iranian languages.

8. 'black': Parachi: Etymology is unknown. Looks like a borrowing. Phonetic variants: pádu, p’adú (Efimov 2009: 225).

9. 'blood': Ormuri: Morgenstierne thinks that Kan. sun Log. šun is borrowed from Indic languages; he compares Sanskrit śona- 'red, crimson, purple / redness / blood'. This is rather uncertain, because this word has later Indic cognates only in Pali sōnita and Prakrit sọnia (Turner 1962-1985: 12625). The correspondence Kan $s \sim \log$. ̌̌, usual for genuine Ormuri words, is also problematic. I would rather hesitantly derive Ormuri sun from PI * $x$ rū- 'raw flesh' with the suffix -na-, compare Avestan $x r \bar{u}$ - 'raw flesh', xrūniia- 'Bluttat, blutige Misshandlung' (Bartholomae 1904: 539). Log. in is known only from one Logar informant. Morgenstierne considers this word to be a borrowing from Pashto win; indeed, there are dialectal forms without $w$ - (Hallberg 2004: 22).

11. 'breast': Proto-Iranian: *war-ah-, found in Avestan and some other languages (for example, Balochi), has a parallel in Old Indian úras- 'breast'. *cai-na- is attested in Southwestern and Northwestern languages.

Ormuri: Log. sónok sínak shows polysemy 'breast / udder'. A dubious case: this word can designate only woman's breast. Distinct from Kan. Log. čik pl. čiči 'nipple / female breast (pl.)' (Efimov 2011: 270; Morgenstierne 1929: 390). Hallberg cites Kan. sina that seems to be borrowed from Persian (Hallberg 2004: 83).

12. 'burn': Proto-Iranian: *dag- is retained in this or slightly altered meaning in Avestan, middle Iranian and some modern Iranian languages. The second candidate *sauk- / ${ }^{*}$ sauč-, widespread in Iranian languages, has the original meaning 'to shine'.

${ }^{*} \theta a w$ - is an Eastern isogloss (mostly Pamir languages).

14. 'cloud': Proto-Iranian: There are two Iranian stems, *abr-a- and *maig-a-, both attested in Avestan and all Iranian subgroups and having external cognates. It seems that the main word for 'cloud' in Young Avestan was maē $\gamma a-$-, while aßra- had the meaning 'rain cloud'. 
However, in other Iranian languages descendants of both these stems can mean 'cloud' in general. Therefore, it is reasonable to think that *abra- and *maiga- were semantically very close to each other in Proto-Iranian, and we have to treat them as synonyms.

15. 'cold' (adj.): Proto-Iranian: *stāba- is an Eastern Iranian isogloss.

18. 'dog': Proto-Iranian: *cwan- has Indo-Aryan cognates, a perfect Indo-European etymology and wide distribution. ${ }^{*} k u t a-/$ fem. ${ }^{*} k u t i(\bar{l})$ - is widespread mostly in eastern Iranian

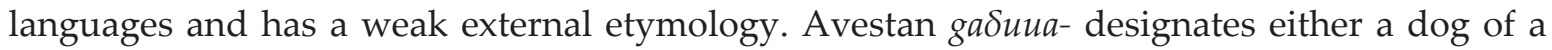
special breed or serves as a stylistic synonym; descendants of this root in other Iranian languages often mean 'puppy' (Ossetic, Yaghnobi) or 'a multicolored dog; a dog with a white mark' (Wakhi), 'a dog with cut ears' (Shughni).

19. 'drink': Proto-Iranian: *hwar- features polysemy 'to eat / to drink' in Avestan, Southwestern languages (Middle Persian, Modern Persian etc.), Northwestern languages (Balochi), Eastern languages (Sogdian, Yaghnobi), Ormuri. Such distribution suggests that *hwar-could be polysemous already in Proto-Iranian.

The second candidate is the root ${ }^{*} p \bar{c}-/{ }^{*} p \bar{a}$ - whose antiquity is proved by Indo-Aryan cognates. This root forms the verbs 'to drink' in some languages of the Pamir group (Wakhi puv-, pov- / pit-, Ishkashimi pbv- / pbvd-, Sanglechi pöv- / pöv- 'to drink'). At the current stage of research it is hard to determine whether the Pamir languages possess a retention or represent a backward development.

22. 'earth': Ormuri: Log. búma bóma, Kan. búma bumba mostly mean 'earth as opposed to heaven / ground', but can also designate 'earth (soil)', compare "after this the seeds are sown (lit. 'scattered on the earth')" (Efimov 2011: 234).

G. Morgenstierne translates Log. jer as 'clay'; moreover, it can be tentatively traced back to *grai- / "gri- 'clay' (EDIL 3: 83). However, it is used in the following contexts: "then for one month (they are occupied) with ditches and channels (to retain water), they take earth (soil, clay), they make vegetable patches (a kord is a plot of ground banked up) - they put earth on the plot"; "the month of Jawza has come - they take earth, put it on the plots, they water them in the spring" (Efimov 2011: 250).

26. 'fat': Proto-Iranian: * $\bar{p}$-wah- is retained in Avestan and many middle and modern Iranian languages from all Iranian subgroups, and has reliable Indo-Aryan and Indo-European cognates. * čarp-īh-is a Western Iranian isogloss. *wāzdā- is an Eastern Iranian isogloss.

29. 'fish': Proto-Iranian: the word ${ }^{*} m a c y-a-\left(<{ }^{*} m a t s y-a-\right)$ is retained in Avestan, Southwestern (middle and modern Persian), Northwestern Iranian languages (Parthian, Kurdish etc.) and in dialects of Pashto; moreover, it has clear Indo-Aryan cognates.

Eastern Iranian *kapa- 'fish' does not occur in other subgroups and has no reliable etymology.

30. 'fly' (v.): Proto-Iranian: *pat- still preserves its original meaning in several Avestan passages, the direct Indo-Aryan cognates speak in favor of its antiquity. In the majority of Iranian languages it was superseded by verbs with different semantics (often prefixed) or denominatives from *par-na- 'feather'.

31. 'foot': Parachi: Polysemy: 'foot / paw'. It is possible to suspect polysemy 'foot / leg', compare 'this woman is pregnant', literally, 'this woman has heavy legs' (Efimov 2009: 160).

Ormuri: Polysemy: 'foot / leg'. The Kaniguram form has an Indian suffix. Hallberg records langay \{lıngai\} (Hallberg 2004: 85).

34. 'good': Proto-Iranian: *wah- $u$ - is retained in Avestan and some other Iranian languages (among them, Khotanese); it has direct Indo-Aryan and Indo-European cognates.

35. 'green': Proto-Iranian: *3ar-i- shows polysemy 'green / yellow'; these meanings are found in Avestan and coincide with the Old Indian situation. In the majority of Iranian lan- 
guages, this adjective with the meaning 'green' was superseded by *sabz- $a$ - 'green' of unclear origin. *axšaina- is an Eastern isogloss.

36. 'hair': Proto-Iranian: *warc- $a$ - is retained in this meaning in Avestan, in Northwestern Iranian Languages (Pahlavi) and in several Eastern Iranian idioms of different periods (for example, Khotanese, Sogdian and Pashto). In Western Iranian it has altered meanings, e.g., Persian gors 'curl'. *maud-a- 'hair' is found only in Southwestern and Northwestern Iranian languages of Middle and Modern periods. The stem *gaun-a-designating 'hair' in Eastern Iranian languages originally meant 'body hair (of animals) / color (of horse)'.

39. 'hear': Proto-Iranian: ${ }^{*} \mathrm{craw}$ - is retained in its basic meaning 'to hear' in Avestan, Balochi and Eastern Iranian languages (the Shughni group and Yazghulami); other Iranian languages of all subgroups preserve only derived meanings, like 'to sing', 'to be heard'. Verbs with the root *gawš- (mostly prefixed) are also attested as 'to hear' in many Iranian languages belonging to all subgroups. However, Avestan gaoš- means 'to listen / to perceive' and Old Indian ghoṣ- means 'to sound'. Therefore, it is unnecessary to treat ${ }^{*}$ craw- and ${ }^{*}$ gauš- as Proto-Iranian synonyms.

43. 'kill': Proto-Iranian: the root "gan- shows polysemy 'to hit / to strike / to slay / to kill'. Retained in Avestan and all Iranian subgroups; has direct Indo-Aryan and IE cognates. In many Iranian languages, it preserves only the meanings 'to hit / to strike', but it is the main word for 'to kill' in Avestan, Parachi, Ormuri and many Eastern Iranian languages (Khotanese, Shughni, Ishkashimi etc.). In Northwestern and Southwestern Iranian languages the main verb with this meaning is *kauš-.

45. 'know': Proto-Iranian: The opposition *wid- 'to know (a situation), know that...' / *zan'to know how to, be acquainted with an object/person' is found in Avestan and can be reconstructed for Proto-Iranian (the same is attested in Old Indian). In the majority of Iranian languages, *wid- 'to know (a situation), know that...' in its primary meaning was superseded by ${ }^{*} z a n-$. In some languages, the meaning 'to know' is expressed with such verbs as * grab- 'to grab / seize / take' etc.

46. leaf: Parachi: $p \bar{n} n$ has the polysemy: 'feather / leaf'.

Ormuri: Kan. puxay shows polysemy 'leaf / feather'. Also Log. paṭ (Morgenstierne 1929: 389), probably borrowed from Dardic languages; compare Pashai pațā 'leaf'.

47. 'lie': Proto-Iranian: * cai- is retained in Avestan and has external cognates. Descendants of this root in later Iranian languages have different meaning: 'to sleep' (Wakhi), 'to be seek, ill' (Ossetic). In the majority of Iranian languages the term 'to lie' is derived from verbs with original meanings 'to sleep', 'to fall' etc.

54. 'moon': Ormuri: Kan. spožmay (Efimov 2011: 272; Hallberg 2004: 92). It is clear from texts in Efimov 2011 and from Hallberg's list that now this borrowing from Pashto has superseded the original maryok in Kaniguram dialect.

55. 'mountain': Proto-Iranian: *gar-i- is retained in this meaning in Avestan, Southwestern, Eastern languages and Ormuri. In Northwestern languages this root is preserved only in altered meanings (compare Kurdish gir, girik 'hill / height').

*kaufa- has reflexes in Persian and Kurdish and is a Western isogloss.

56. 'mouth': Proto-Iranian: *āh- is retained only in Avestan and Khotanese, but its antiquity is proven by direct Indo-Aryan and IE cognates. *3af-an-is used with this meaning in Southwestern and Northwestern Iranian groups. The original meaning may have been 'muzzle' (in Avestan - 'mouth of daevic creatures').

58. 'neck': Proto-Iranian: * grīw- $a$ - is retained in Avestan, Southwestern and Eastern Iranian languages, its antiquity is supported by direct Indo-Aryan cognates. The widespread stem *gard-a-na- *gart-a-na- 'round' with the semantic development 'round' > 'neck' represents a later derivative from *gart- * gard- 'to turn'. 
61. 'nose': Proto-Iranian: *nah- / *nāh- is retained in Avestan, Old Persian and all subgroups and has direct Indo-Aryan cognates. The stem *wain-a- * wain-i-, derived from *wain'to see', means 'nose' in some Iranian languages mostly of Southwestern and Northwestern subgroups. In Avestan it means 'nose of dragon'. We can treat it as a Western isogloss.

Ormuri: Kan. Log. ninní can represent a contamination of *nah- / ${ }^{*} n a: h$ - and *wain-i-.

66. 'red': Proto-Iranian: * raud-i-ta- is retained in Avestan and has direct Indo-Aryan and IE cognates. The stem *suk-ra- *sux-ra-, derived from the root *sauč- 'to shine', is widespread in Iranian languages with the meaning 'red', but its original semantics should be 'shining, bright; red (of fire)'.

67. 'road': Proto-Iranian: *pant- $\bar{a}-\sim{ }^{*} p a \theta$ - is a basic term in Avestan and mostly in Eastern languages (in Middle and modern Southwestern and Northwestern languages it means specifically 'path / track / direction / advice, counsel'). Cf. the stem ${ }^{*} r a \theta-y a$, which acquires the semantics 'road' in Western Iranian languages and Pashto, being derived from the word * $r a \theta a-$ 'chariot'.

68. 'root': Proto-Iranian: both *waix-a- and *raiš-a-ka- are retained in all subgroups (sometimes they represent synonyms within one language). These stems do not have certain external cognates. We treat them as synonyms.

70. 'sand': Proto-Iranian: a very unstable term. One of the possible candidates is *rayka-, attested in Southwestern and Northwestern Iranian languages.

71. 'say': Proto-Iranian: suppletive roots *mraw- and *wač- are the main terms with the meaning 'to say' in Avestan; the first one is used in present, the second one mostly in the aorist and perfect. Old Indian data confirm that Avestan retains the original suppletive paradigm. * gaub-in this meaning represents a Western isogloss (EDIL 3: 232).

72. 'see': Proto-Iranian: *wain- forms the present stem. *darc- is used as perfect and aorist stem in Avestan and as past stem in Parachi and some Eastern Iranian languages. The third stem *dāi- usually forms the past stem in all subgroups, but Indo-Aryan cognates demonstrate another meaning.

Both Parachi and Ormuri have suppletive paradigms with preservation of *darc-. The stem *wain- yields Kan. dzun- (Efimov 2011: 283).

74. 'sit': Proto-Iranian: according to Avestan and external Indo-Aryan data, Proto-Iranian *āh- meant 'to sit' and *had- meant 'to sit down'. 'a $\bar{h}$ - is retained for the stative meaning 'to sit' in Avestan and Middle Iranian languages. In the majority of Iranian languages, belonging to all subgroups, *had-acquired the meaning 'to sit', having superseded *āh-.

Ormuri: Compare the phrase \{a-dúwa-wa mux-ki āyi aw řawa bu\} 'her daughter sits in front of her and cries'. The present stem $\bar{a} y$ - ey- is distinct from related Kan. $n$ - (2nd conj.) nay- Log. ney- 'to sit down' with the same participle Kan. nás-tak Log. nós-tok (Efimov 2011: 291-293; Morgenstierne 1929: 401).

83. 'swim': Proto-Iranian: the verb *fraw- is retained in the meaning 'to swim' only in some Avestan texts, but direct Indo-Aryan and IE cognates prove its antiquity. In later Iranian languages, it was superseded by various other verbs or analytic expressions.

85. 'that', 86. 'this': Proto-Iranian: On the basis of Avestan data, the Proto-Iranian system can be tentatively reconstructed as *ai- (masc.) *i- (fem., neut.) 'this' / *ana- 'that' (medial) / *haw- (masc., fem.) *awa- (neut.) 'that' (distal).

90. 'tree': Proto-Iranian: ${ }^{*} d \bar{a} r-u$ - 'tree / wood / stick' is retained in all subgroups. It or its derivatives mean 'tree' in Southwestern and Northwestern Iranian languages. In light of external comparanda, * $d \bar{a} r-\mathcal{u}$ - can be safely posited as a Proto-Iranian term for 'tree'. Thus it is likely that *dār-u- in the meaning 'tree' was superseded with *wan-a- 'forest / (forest) tree' already in Avestan and in the Eastern Iranian group. 
92. 'walk (go)': Proto-Iranian: the root *ai- is retained in Avestan and all subgroups. It forms both present and aorist stems in Avestan. In many languages of all subgroups descendants of *cyaw- 'to set in motion; to move' became the main verbs with the meaning 'to go'.

93. 'warm (hot)': Proto-Iranian: *gar-ma- 'hot / warm' is retained in Avestan and all subgroups; it has direct Indo-Aryan and IE cognates. *tap- forms words with this meaning in Eastern Iranian languages.

Ormuri: Kan. Log. tok shows polysemy 'hot / warm'.

104. 'salt': Proto-Iranian: Kurdish $x w \bar{e}$ 'salt' and Balochi $v \bar{a} d$ are derived from *hwād- 'delicious; to be delicious'.

105. 'short': Proto-Iranian: ${ }^{*} m r 3-u$ - is retained in Avestan (in the temporal sense), Khotanese and Sogdian. External comparanda prove its antiquity.

106. 'snake': Proto-Iranian: ${ }^{*} a \breve{z}-i$ - is retained in Avestan, in Middle Persian in the meaning 'dragon', in Khwarezmian and Munji; this stem has Indo-Aryan and IE cognates and this situation proves that it should be considered as the main Iranian word for 'snake'. *mār-a-, probably derived from *mar- 'to kill', serves as a designation of 'snake' in Southwestern and Northwestern Iranian languages. In many Iranian languages, especially Eastern, descendants of ' $k r m-i$ - 'worm' altered the meaning to 'snake' (or the polysemy 'worm / snake' can be found). Eastern languages also have one more stem whose reconstruction is uncertain, yielding Shughni divüsk, Sarikoli tolfolsk, Wakhi fuks 'snake' etc.

107. 'thin': Proto-Iranian: * $n \bar{a} z-u-k a-$, originally meaning 'tender', replaced this meaning in some Southwestern and Northwestern languages.

110. 'year': Proto-Iranian: according to Avestan data and IE cognates, *yār-meant 'year' in Proto-Iranian. *car-da- with the original Proto-Indo-Iranian meaning 'autumn' alters it to 'year (of somebody's age)' in Avestan and to 'year (in general)' in later Iranian languages.

\section{Abbreviations of language names}

Arab. - Arabic; Dard. - Dardic; EIr - Eastern Iranian; Ind. - Indian; Ir. - Iranian; Kan. - Kaniguram Ormuri; Log. - Logar Ormuri; OInd. - Old Indian; Pers. - Persian (Dari); Psht. - Pashto; SW - Southwestern Iranian; NW - Northwestern Iranian; PIr - Proto-Iranian.

\section{References}

Bartholomae, Christian. 1904. Altiranisches Wörterbuch. Strassburg: Verlag von Karl. J. Truebner.

Baranov, Kh. K. 1977 Arabsko-russkij slovar'. 5th edition. Moskva: Russkij jazyk.

Cheung, Johnny. 2007. Etymological dictionary of the Iranian verb. Leiden-Boston: Brill.

Grierson, George A. 1918. The Ōrmuṛī or Bargistā language. An account of a little-known Eranian dialect. Memoirs of the Asiatic Society of Bengal 7/1: I-XIV, 1-101.

Dybo, Anna V., George S. Starostin. In Defense of the Comparative Method, or the End of the Vovin Controversy. In: I. Smirnov (ed.). Aspekty komparativistiki III. Orientalia et Classica: Trudy Instituta vostochnykh kul'tur i antichnosti XIX: 119-258. Moskva: RGGU.

Dybo, V. A. 1974. Afganskoe udarenie i ego znachenie dl'a indoevropejskoj i balto-slav'anskoj akcentologii. I. Imennaja akcentuacija. In: T. M. Sudnik (ed.). Balto-slav'anskie issledovanija: 67-105. Moskva: Nauka.

Dybo, V. A. 1989. Afganskoe udarenie i ego znachenie dl'a indoevropejskoj i balto-slav'anskoj akcentologii. II. Glagol'naja akcentuacija. In: N. I. Tolstoj (ed.). Slav'anskij I balkanskij fol'klor. Prosodija: Sbornik statej: $106-147$. Moskva: Nauka.

EDIL = V. S. Rastorgueva, D. I. Edelman. 2000-. Etimologicheskij slovar' iranskikh jazykov (Etymological dictionary of the Iranian languages). Moskva: Vostochnaja literatura RAN. 
Efimov, V. A. 1986. Jazyk ormuri v sinkhronnom i istoricheskom osveschenii. Moskva: Nauka.

Efimov, V. A. 2009. Jazyk parachi. Moskva: Vostochnaja literatura.

Efimov, V. A. 2011. The Ormuri language in Past and Present. English translation edited by Joan L. G. Baart. FLI language and culture series (Volume 6). Forum for Language Initiatives.

Hallberg, Daniel G. 2004. Sociolinguistic survey of Northern Pakistan. Volume 4. Pashto. Waneci. Ormuri. $2^{\text {nd }}$ edition. Islamabad: National Institute of Pakistan Studies, Quaid-i-Azam University and Summer Institute of Linguistics.

Kieffer, Charles M. 1989. Le parāčī, l'ōrmuṝi. In: R. Schmitt (Hrsg.) Compendium linguarum Iranicarum: $445-455$. Wiesbaden: Dr. Ludwig Reichert Verlag.

Kieffer, Charles M. 2009. Parachi. In: G. Windfuhr (ed.). The Iranian languages: 693-720. London / New York: Routledge.

Mayrhofer, Manfred. 1989. Vorgeschichte der iranischen Sprachen; Uriranisch. In: R. Schmitt (Hrsg.) Compendium linguarum Iranicarum: 445-455. Wiesbaden: Dr. Ludwig Reichert Verlag.

Morgenstierne, Georg. 1926. Report on a linguistic mission to Afghanistan. Oslo: H Aschehoug \& Co.

Morgenstierne, Georg. 1929. Indo-Iranian frontier languages. Volume I. Parachi and Ormuri. Oslo: H Aschehoug \& Co. Morgenstierne, Georg. 1974. Etymological Vocabulary of the Shughni group. Wiesbaden: Dr. Ludwig Reichert Verlag.

Morgenstierne, Georg. 2003. A new etymological vocabulary of Pashto. By Georg Morgenstierne; compiled and edited by J. Elfenbein, D.N. MacKenzie and Nicholas Sims-Williams. Wiesbaden: Dr. Ludwig Reichert Verlag.

Oranskij, I. M. 1979. Vvedenie. Voprosy istoriko-dialektologicheskoj klassifikacii iranskikh jazykov. In: V. S. Rastorgueva (ed.). Osnovy iranskogo jazykoznanija. Dreoneiranskie jazyki: 81-128. Moskva: Nauka.

Rees, Daniel A. 2008. Towards Proto-Persian: An Optimality Theoretic Historical Reconstruction (Ph.D.). Washington, DC.

Starostin, George S. 2016. From wordlists to proto-wordlists: reconstruction as 'optimal selection'. Faits de langues 47: 177-200.

Starostin, Sergei (ed.). 1998-2005. The Tower of Babel. An etymological database project. Available at: http://starling.rinet.ru/ [accessed 26.12.2018].

Tedesco, Paul. 1921. Dialektologie der west-iranischen Turfantexte. Le Monde oriental 15: 184-258.

Turner, Ralph L. 1962-1985. A comparative dictionary of Indo-Aryan languages. London: Oxford University Press. Available at: http://dsal.uchicago.edu/dictionaries/soas/ [accessed 26.12.2018].

А. А. Трофимов. О классификации языков парачи и ормури внутри иранской группы по данным аннотированных сводешевских списков

В данной статье рассматриваются языки парачи и ормури и их место в иранской классификации. На основании данных сопоставления двух аннотированных 110-словных списков Сводеша, составленных для этих языков, с реконструированным праиранским списком и западноиранскими и восточноиранскими регионализмами, делается вывод о более вероятном восточноиранском характере парачи и ормури - эти языки имеют совпадения с восточноиранскими изоглоссами и практически не имеют совпадений с многочисленными западноиранскими. При этом показательно, что фонетические изоглоссы не дают ясной картины: у парачи и ормури есть как западные, так и восточные фонетические черты, причём интересно, что акцентуационные системы этих языков напоминают систему, засвидетельствованную в пушту и в наибольшей степени отражающую древнее (индо)иранское состояние. Таким образом, более обоснованно мнение Г. Моргенстьерне о принадлежности парачи и ормури к восточноиранским языкам. При этом выделение норвежским исследователем отдельной юго-восточной иранской подгруппы, единственными представителями которой и являются рассматриваемые языки, спорно и нуждается в дальнейшем подтверждении.

Ключевые слова: парачи, ормури, 110-словный список Сводеша, праиранский язык, восточноиранские языки, западноиранские языки, фонетические изоглоссы, акцентуационные системы, пушту. 